Baltic Astronomy, vol.1, 545-559, 1992.

\title{
A POSSIBILITY OF PHOTOMETRIC CLASSIFICATION OF STARS WITH THE WIDE FIELD CAMERA OF THE HST
}

\author{
V. Straižys and G. Valiauga \\ Institute of Theoretical Physics and Astronomy, \\ Goštauto 12, Vilnius 2600, Lithuania
}

Received April 20, 1992.

\begin{abstract}
A combination of seven filters contained in the Wide Field and Planetary Camera of the Hubble Space Telescope makes it possible to realize a photometric system similar to the Vilnius system and capable to classify photometrically stars of all spectral types in two or three dimensions (temperature, luminosity and metallicity or peculiarity) in the presence of interstellar reddening. It is suggested to join the close bandpasses of the most popular medium-band photometric systems and to place the optimum filters into one of the next generation WF cameras of the HST. The system will then allow to classify extremely faint stars in two or three dimensions both in our Galaxy and in other galaxies.
\end{abstract}

Key words: Hubble Space Telescope - photometric systems - stellar classification.

\section{The Wide Field Camera}

The Wide Field Camera of the Hubble Space Telescope was intended for a wide variety of two-dimensional imaging astronomical observations. It is possible to change the focal ratio of the camera

Send offprint requests to: V. Straižys 
from $f / 12.9$ to $f / 30$. With this second focal ratio the camera is intended for obtaining high-resolution planetary images and as a result the whole instrument is usually called the Wide Field and Planetary Camera (WF/PC). Here we shall discuss only the WF part of the camera. It gives a $2.7 \times 2.7$ arcmin field on the detector consisting of a mosaic of four CCD arrays of $800 \times 800$ pixels each. The pixel size corresponds to 0.1 arcsec. The camera now operating is equipped with 48 light filters covering a wide range of wavelengths (1150$11000 \AA$ ) and halfwidths. The camera and its filters are described in detail by Westphal (1982), Griffiths $(1985,1990)$ and MacKenty et al. (1992).

Originally the camera was intended to achieve sensitivity of better than $V=28 \mathrm{mag}$ for point sources at one hour exposure. However, due to spherical aberration of the main mirror of the HST, the limiting magnitude of the Wide Field Camera is about 2-3 mag lower. This limiting magnitude is within the capabilities of the largest ground-based telescopes working in uncrowded fields. However, the main advantage of the HST relative to telescopes on the ground is that it can work in more crowded regions (Holtzman et al., 1991).

At the same time a very low accuracy of the WF camera photometry has been achieved. Hughes (1992) reports the accuracy of the order of $5-10 \%$.

Plans are underway for replacement of the original WF/PC with a second generation camera WFPC-2. The new camera will be fitted with corrective optics for compensating spherical aberration of the optical system of the telescope. About half of filters will be replaced.

The original filter set of the WF/PC has been severely critisized since, when selecting the filters, almost all existing standard photometric systems were ignored. Even the $U B V R I$ filters are very far from the standard system. Transformation of the WF/PC colour indices into the standard systems is very complicated (Koornneef et al., 1986; Edvardsson and Bell, 1989; Harris et al., 1991; Paltoglou and Bell, 1991) and this prevents using the ground-based photometric sequences in the standard systems for calibration of the space photometry. The WFPC-2 photometric filter set is without serious changes, except of inclusion of the $v, b$ and $y$ filters of the Strömgren photometric system (Trauger, 1989; Trauger and Brown, 1992). On several occasions one of us has suggested to realize in the WF camera the Vilnius photometric system but with no reaction from the HST specialists. What are arguments in favour of this system? 


\section{The Vilnius photometric system}

At the beginning of the seventies a number of medium-band photometric systems were introduced for photometric classification of stars which are too faint for their spectral analysis. Among them was the Vilnius photometric system consisting of seven medium bandpasses listed in Table 1.

Table 1. Mean wavelengths and half-widths of the Vilnius photometric system

\begin{tabular}{lrrrrrrr}
\hline & $U$ & \multicolumn{1}{c}{$P$} & \multicolumn{1}{c}{$X$} & \multicolumn{1}{c}{$Y$} & \multicolumn{1}{c}{$Z$} & \multicolumn{1}{c}{$V$} & \multicolumn{1}{c}{$S$} \\
\hline$\lambda_{0}(\mathrm{~nm})$ & 345 & 374 & 405 & 466 & 516 & 544 & 656 \\
$\Delta \lambda(\mathrm{nm})$ & 40 & 26 & 22 & 26 & 21 & 26 & 20 \\
\hline
\end{tabular}

The system has been described in English by Straižys and Sviderskiene (1972) and Straižys (1973, 1992) and in Russian by Straižys (1977). Selection of bandpasses of the system for the first time has been based on quantitative analysis of detailed energy distribution functions which appeared at the beginning of the sixties. The newest interstellar dust transmission curve was also used. The $U$ bandpass measures intensity beyond the Balmer jump. The $P$ bandpass measures absorption of the higher members of the Balmer series which is sensitive to surface gravity in B-A-F stars. The $X$ bandpass measures intensity before the Balmer jump for early-type stars as well as the amount of blocking by metallic lines for late-type stars. The $Y$ bandpass is placed near the break-point of the interstellar extinction law and is very important in distinguishing the temperature and interstellar reddening. The $Z$ bandpass is placed on the wide absorption feature formed by the $\mathrm{MgI}$ triplet lines and a $\mathrm{MgH}$ band. The depth of this feature is very sensitive to surface gravity or luminosity of $\mathrm{K}-\mathrm{M}$ stars. The $V$ bandpass is a mediumband analogue of $V$ in the $U B V$ system. The $S$ bandpass is placed on the $\mathrm{H} \alpha$ line and plays the role of its emission discriminator among early-type stars. Among late-type stars $S$ bandpass measures pseudocontinuum intensity.

The Vilnius system allows one to classify stars of all temperatures in spectral classes and absolute magnitudes and F-G-K stars in metallicities when interstellar reddening is present and when no information is available from stellar spectra. The following peculiar types of stars can be detected: Be, Am, Ap, subdwarfs, metal-deficient giants, carbon and barium stars, Herbig $\mathrm{Ae} / \mathrm{Be}$ and $\mathrm{T}$ Tauri-type 
stars, white dwarfs, horizontal-branch stars and a number of types of unresolvable binaries. Additionally, there is a possibility to classify photometrically galaxies into their morphological types and to estimate their redshifts (Straižys and Sviderskienè, 1983). No other system is capable of solving such a wide variety of classification problems.

The system has been realized with a CCD detector having quantum efficiency $33 \%$ at $350 \mathrm{~nm}$ (Boyle et al., 1990a,b; Smriglio et al., 1991). CCD photometry with exposures in $U$ and $P$ filters of $20 \mathrm{~min}$ and in other filters of 2-4 min on the $90 \mathrm{~cm}$ telescope of Kitt Peak National Observatory yielded MK spectral types down to $V=17$. This means that with ultraviolet exposures of the order of 1 hour and a $2.5 \mathrm{~m}$ telescope stars with $V=20$ are accessible (Straižys, 1992b). From outside the atmosphere much fainter objects can be reached.

Consequently, the use of the Vilnius photometric system is an optimum way to obtain two- and three-dimensional classification of faint stars affected by interstellar reddening. Such classification will be extremely important for the HST objects.

\section{A pseudo-Vilnius photometric system with the HST filters}

The first WF/PC camera which is now in orbit contains the following seven filters with their mean wavelengths close to the Vilnius system filters (Fig. 1). These filters are listed in Table 2.

Table 2. Mean wavelengths and half-widths of the WF/PC filters close to the Vilnius system

\begin{tabular}{lrrrrrrr}
\hline & $F 336 W$ & $F 375 N$ & $F 413 M$ & $F 469 N$ & $F 517 N$ & $F 547 M$ & $F 656 N$ \\
\hline$\lambda_{0}(\mathrm{~nm})$ & 336.0 & 375.5 & 412.5 & 468.7 & 517.0 & 545.4 & 655.9 \\
$\Delta \lambda(\mathrm{nm})$ & 41.1 & 8.2 & 24.8 & 2.7 & 8.7 & 43.8 & 1.9 \\
\hline
\end{tabular}

Only the ultraviolet filter F336, the violet filter F413 and the green filter $F 547$ are sufficiently wide to simulate the bandpasses of the Vilnius photometric system. Other filters are too narrow and, consequently, their limiting magnitude is much brighter. However, it seems to be interesting to see, if it is possible to realize the Vilnius system with these filters. In other words, we decided to calculate colour equations between the indices obtained with the sets of filters listed in Table 1 and Table 2. 


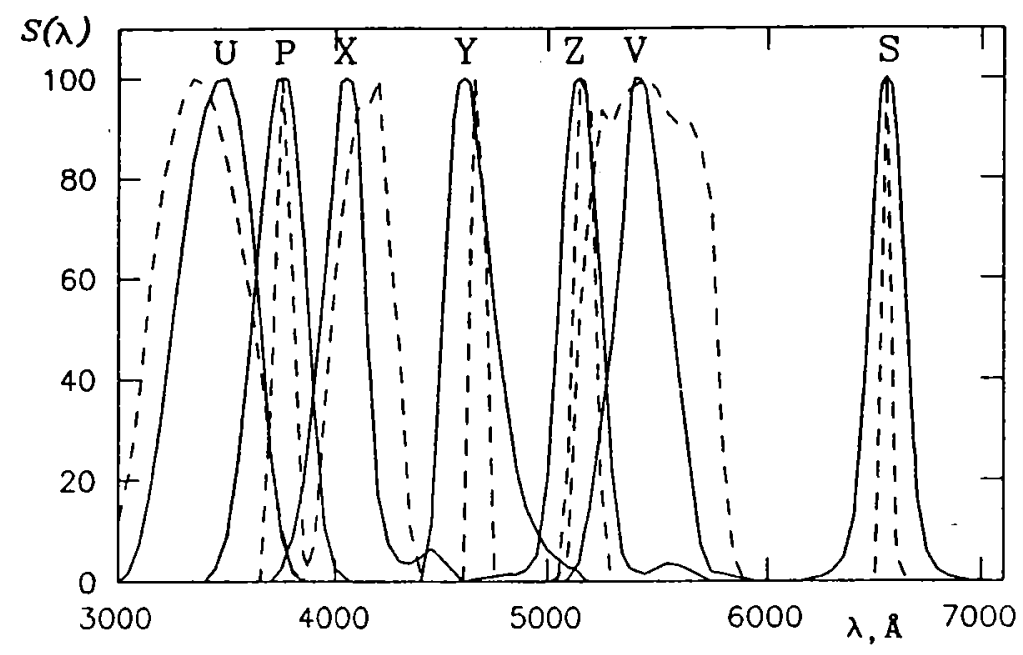

Fig. 1. Response functions of the Vilnius and HST systems.

Synthetic colour indices of both the systems were calculated by numerical convolution using the following equation:

$$
m_{1}-m_{2}=-2.5 \log \frac{\int F(\lambda) S_{1}(\lambda) d \lambda}{\int F(\lambda) S_{2}(\lambda) d \lambda}+2.5 \log \frac{\int F_{0}(\lambda) S_{1}(\lambda) d \lambda}{\int F_{0}(\lambda) S_{2}(\lambda) d \lambda},
$$

where $F(\lambda)$ is the energy distribution function of a star taken from Sviderskiene (1988), $F_{0}(\lambda)$ is the energy distribution function of the O-type star from the same source, $S_{1}(\lambda)$ and $S_{2}(\lambda)$ are the response functions of two magnitudes forming the colour index $m_{1}-m_{2}$. The response functions of the standard Vilnius system were taken from Straižys and Zdanavičius (1970) and Straižys (1992a). The response functions of the HST system were taken from the HST Wide Field and Planetary Camera Instrument Handbook, Version 2.1 (Griffiths, 1990). Before using in integration, all narrow-band response functions were transformed into histogram with the $5 \mathrm{~nm}$ step, having the center of weight at the mean wavelength of the filter. Both sets of response functions are intercompared in Fig. 1. To determine the interstellar reddening lines, we used the energy distribution function of a star convolved with the interstellar extinction law from Straižys (1992a), i.e. $F(\lambda) \tau^{x}(\lambda)$, where $\tau^{x}(\lambda)$ is the transmission of light by $x$ amount units of interstellar dust. The step of integration, $5 \mathrm{~nm}$, is defined by the character of energy distribution data. 


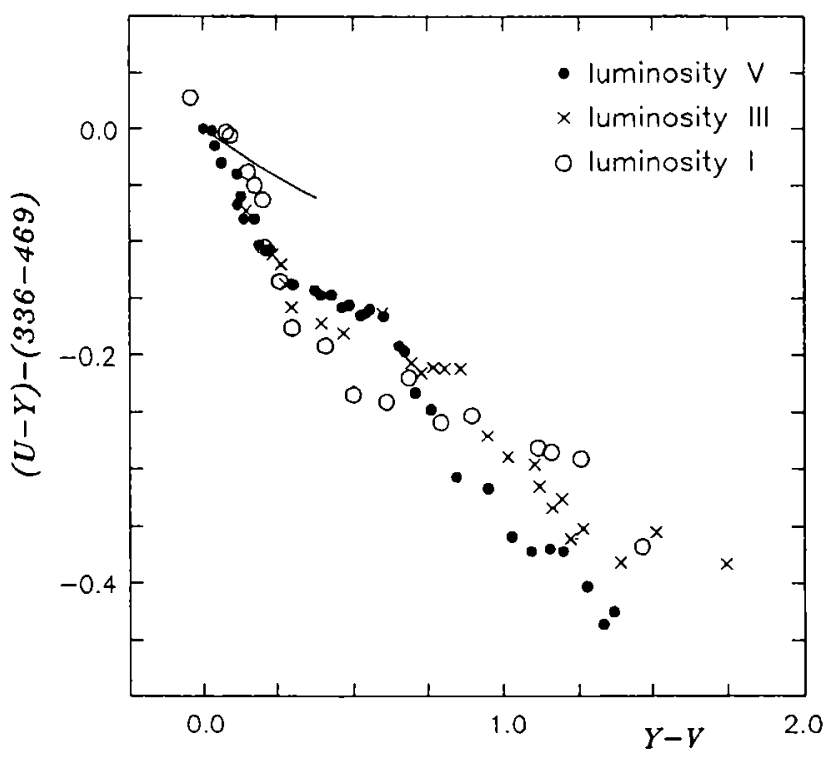

Fig. 2. Difference of the colour indices $U-Y$ and $336-469$ plotted against $Y-V$. The reddening line of the $\mathrm{O}$-type star is also shown.

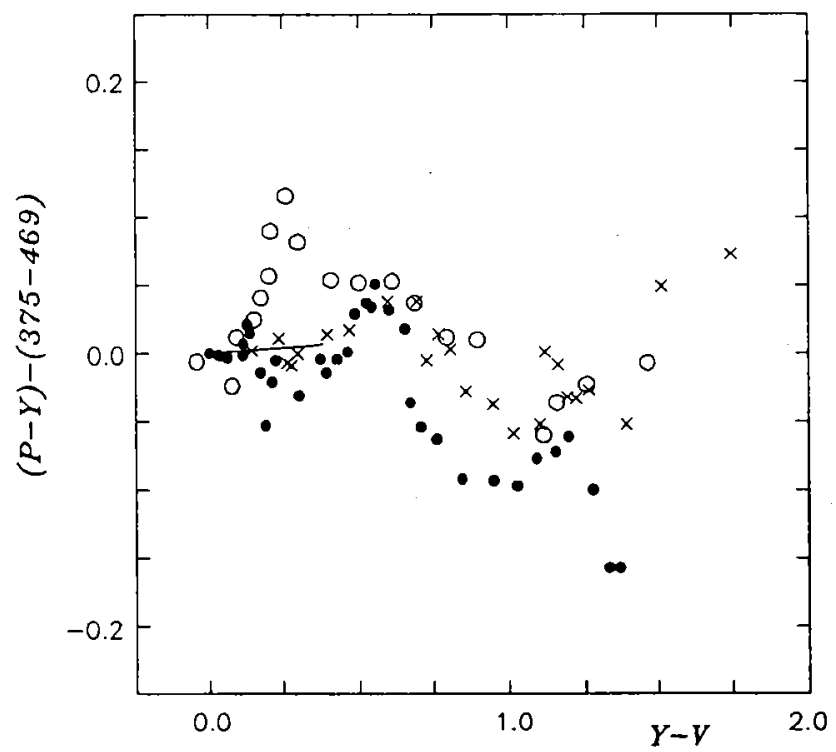

Fig. 3. Difference of the colour indices $P-Y$ and $375-469$ plotted against $Y-V$. The symbols are as in Fig. 2. 


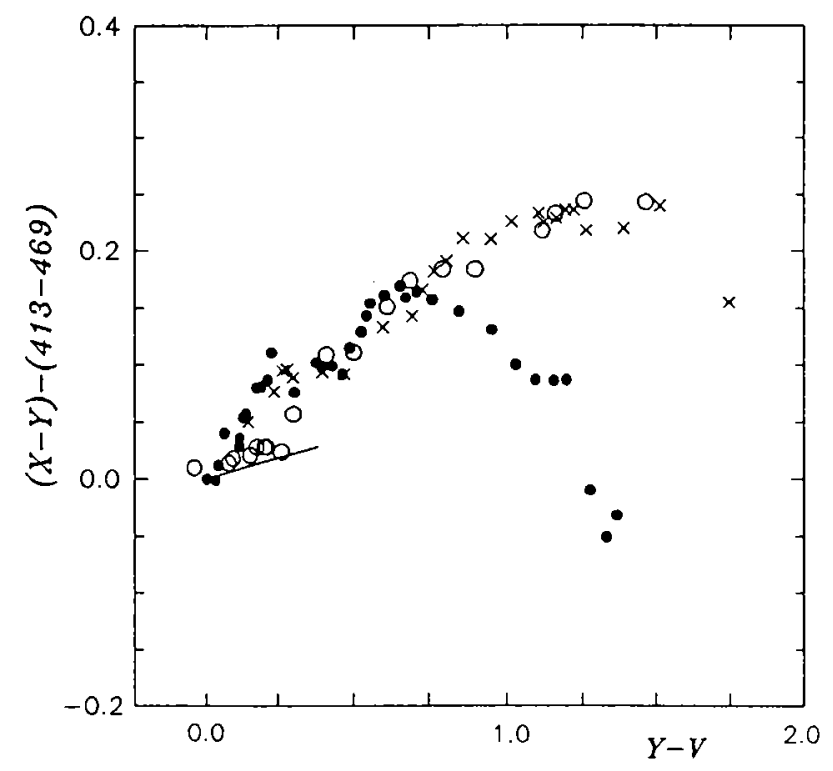

Fig. 4. Difference of the colour indices $X-Y$ and 413-469 plotted against $Y-V$. The symbols are as in Fig. 2.

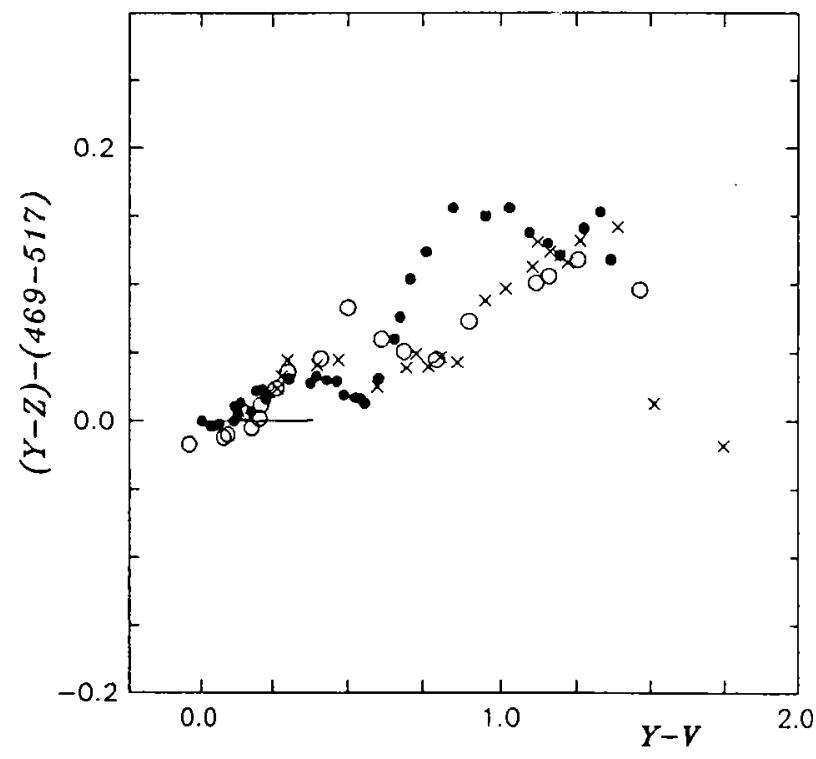

Fig. 5. Difference of the colour indices $Y-Z$ and $469-517$ plotted against $Y-V$. The symbols are as in Fig. 2. 


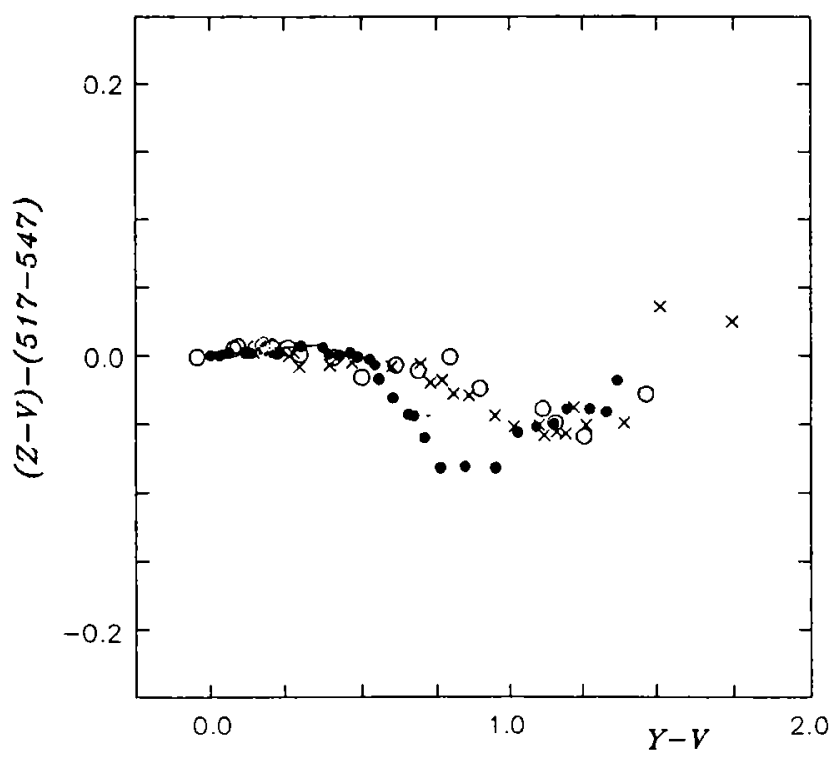

Fig. 6. Difference of the colour indices $Z-V$ and $517-547$ plotted against $Y-V$. The symbols are as in Fig. 2.

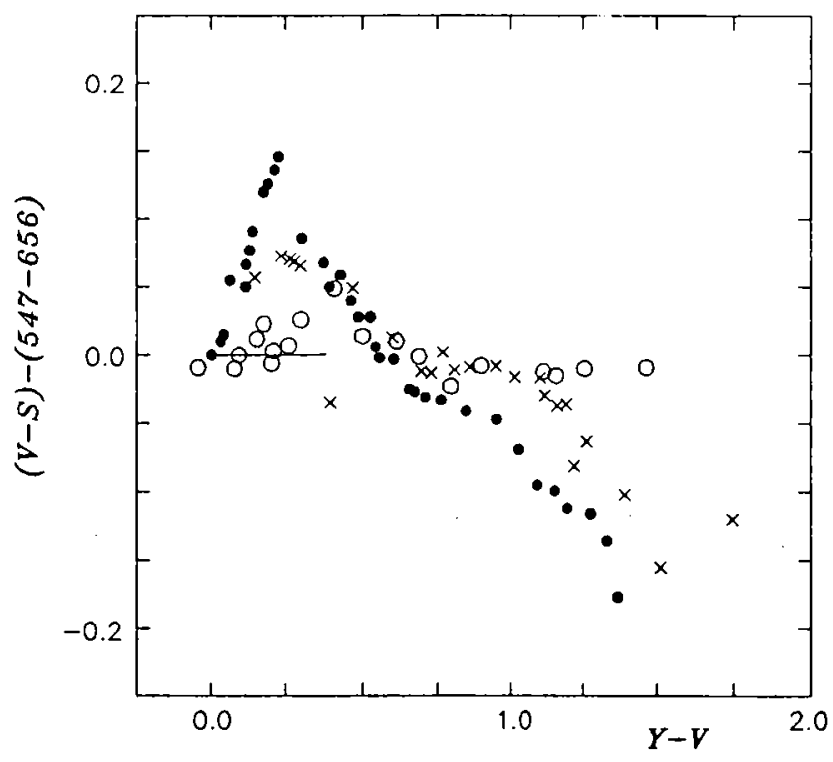

Fig. 7. Difference of the colour indices $V-S$ and $547-656$ plotted against $Y-V$. The symbols are as in Fig. 2. 
Differences of corresponding colour indices of the Vilnius system and the HST system against the colour index $Y-V$ of the Vilnius system are plotted in Figs. 2-7. Dots are main sequence stars, crosses are giants and open circles are supergiants. The reddening line of the O-type star is shown.

One can see that accurate transformation of colour indices from the HST system to the standard Vilnius system is impossible. In all cases sequences of different luminosity classes are nonlinear and do not coincide. Interstellar reddening lines do not coincide with the sequences of unreddened stars as well. Exceptionally large colour equations are observed between the colour indices $U-Y$ and $336-$ 469 , as well as between $X-Y$ and $413-469$. The closest indices are $Z-V$ and $517-547$. However, even in this case a difference exists between the sequences of $\mathrm{G}-\mathrm{K}$ dwarfs and giants.

Thus, there is no possibility to transform colour indices of the HST system into the standard Vilnius system. However, it is interesting to analyse the classification possibilities of the HST system. For this we calculated the interstellar reddening-free $Q$-parameters in the HST system, similar to those $Q$-parameters of the Vilnius system which are in use for classification of stars in spectral types and luminosities: $Q_{336,375,469}, Q_{375,469,547}, Q_{413,469,547}, Q_{413,469,517}$, $Q_{413,517,656}, Q_{336,413,469}$ and $Q_{336,375,469,547}$. Here

$$
Q_{123}=\left(m_{1}-m_{2}\right)-\frac{E_{m_{1}-m_{2}}}{E_{m_{2}-m_{3}}}\left(m_{2}-m_{3}\right) .
$$

Colour excess ratios were calculated for the normal interstellar extinction law as it was described on p. 549 .

$Q, Q$ diagrams, analogues to those which are in use in the Vilnius photometric system, are plotted in Figs. 8-13. The diagrams are plotted only for those types of stars which exhibit the best separation of luminosity sequences. All these diagrams are very similar to their counterparts in the Vilnius system. Thus, if need be, the HST photometric system may be used for two-dimensional classification of faint stars, using the $Q, Q$ diagrams listed in Table 3.

\section{Discussion}

The above results demonstrate that there is no problem to realize in the WF camera of the HST a photometric system capable to classify all types of stars from $B$ to $M$ in the presence of interstellar 


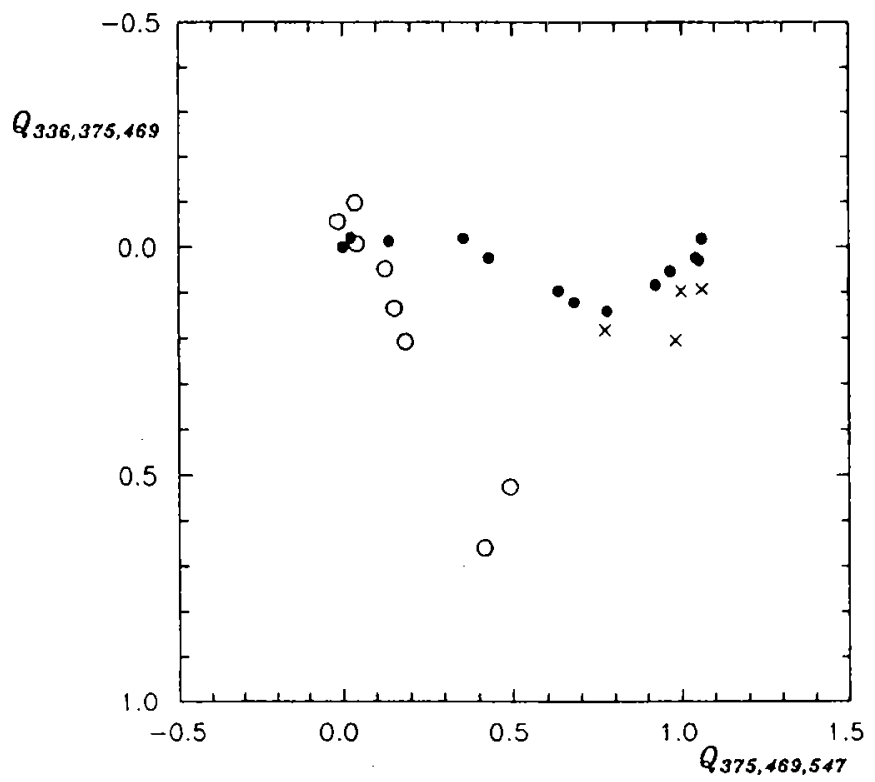

Fig. 8. $Q_{336,375,469}, Q_{375,469,547}$ diagram for classification of $\mathrm{B}$ and A stars. The symbols are as in Fig. 2.

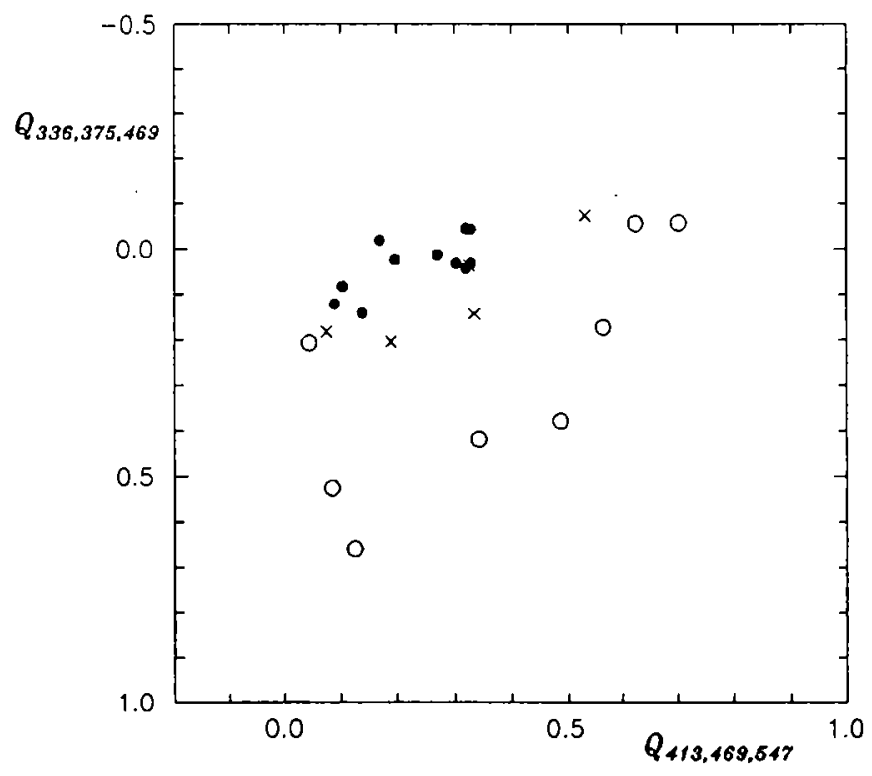

Fig. 9. $Q_{336,375,469}, Q_{413,469,547}$ diagram for classification of B8-A3 and F5-G5 stars. The symbols are as in Fig. 2. 


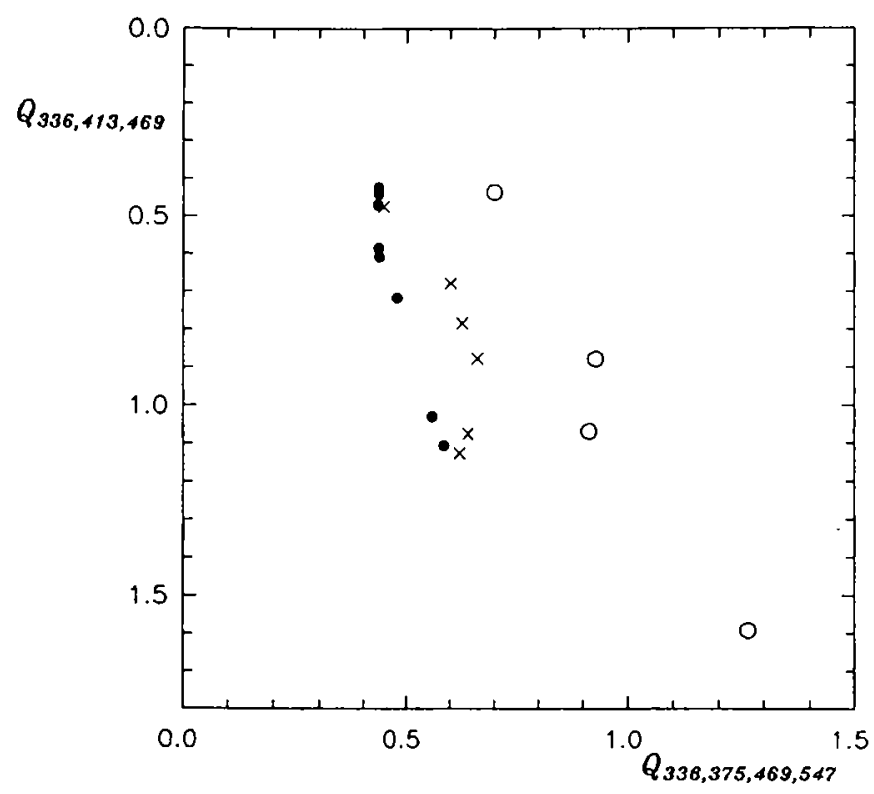

Fig. 10. $Q_{336,413,469}, Q_{336,375,469,547}$ diagram for classification of A5-G0 stars. The symbols are as in Fig. 2.

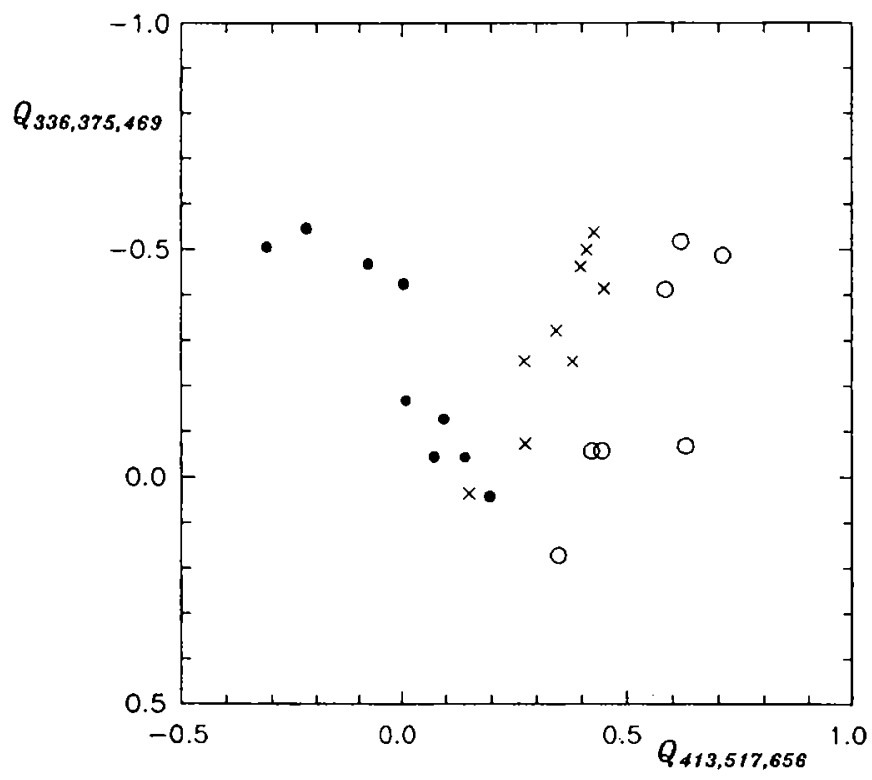

Fig. 11. $Q_{336,375,469}, Q_{413,517,656}$ diagram for classification of G0$\mathrm{K} 5$ stars. The symbols are as in Fig. 2. 


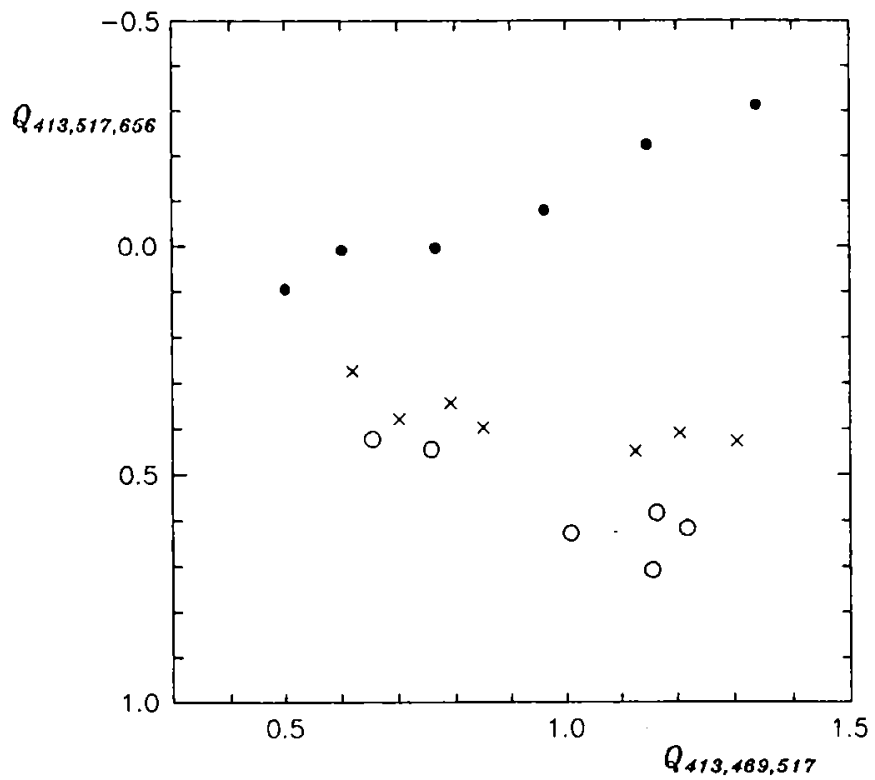

Fig. 12. $Q_{413,517,656}, Q_{413,469,517}$ diagram for classification of G8K5 stars. The symbols are as in Fig. 2.

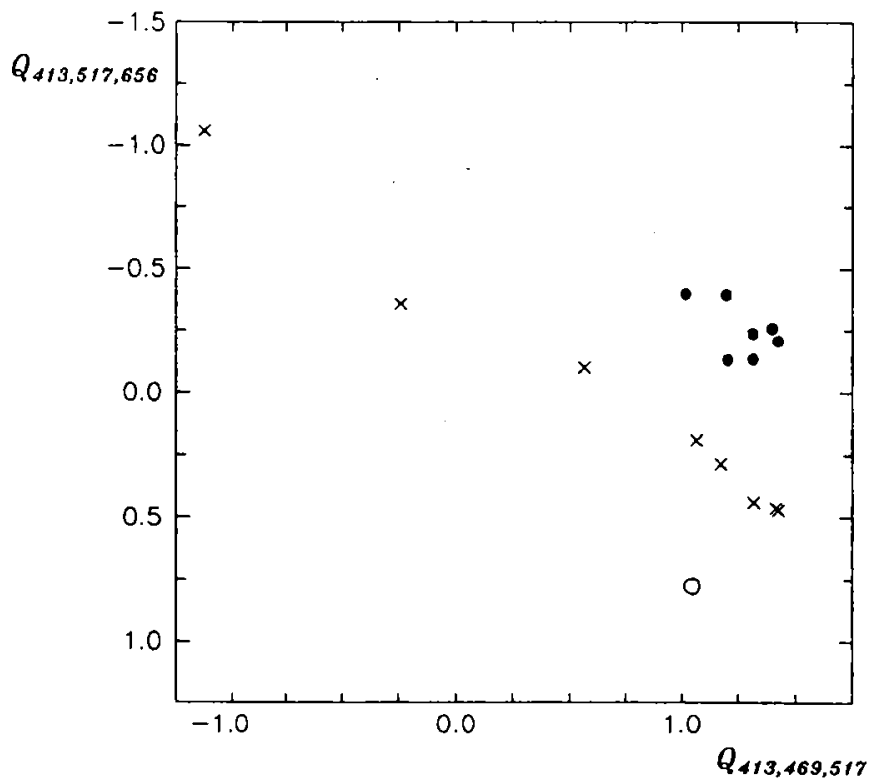

Fig. 13. $Q_{413,517,656}, Q_{413,469,517}$ diagram for classification of M stars. The symbols are as in Fig. 2. 
Table 3. $Q, Q$ diagrams of the HST photometric system useful for twodimensional classification of stars

\begin{tabular}{cc}
\hline$Q, Q$ diagram & Types of stars \\
\hline$Q_{336,375,469}, Q_{375,469,547}$ & B and A \\
$Q_{336,375,469}, Q_{413,469,547}$ & B8-A3 and F5-G5 \\
$Q_{336,413,469}, Q_{336,375,469,547}$ & A5-G0 \\
$Q_{336,375,469}, Q_{413,517,656}$ & G0-K5 \\
$Q_{413,517,656}, Q_{413,469,517}$ & G8-K5 (and M) \\
\hline
\end{tabular}

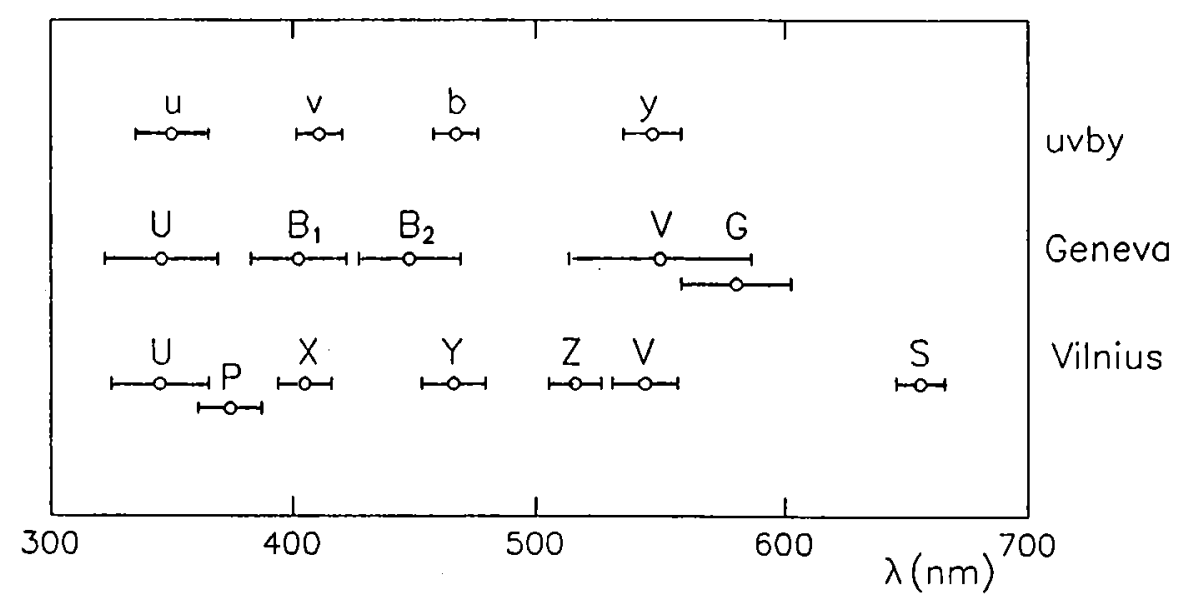

Fig. 14. Comparison of positions and half-widths of response functions of the Strömgren, Geneva and Vilnius systems.

reddening. Certainly, the present system $336,375,413,469,517,547$, 656 contains some narrow bandpasses and this considerably limits the magnitude which can be reached with the HST. However, long exposures, without necessity to take into account the atmospheric extinction, make the classification possible. The use of high quality interference filters having maximum allowed width of transmission curves would give a unique possibility to classify very faint stars both in our Galaxy and in other galaxies.

We understand, that it is a problem how to fit into the filter assembly of the HST the filters of all necessary photometric systems. However, it is not very difficult to solve the problem by uniting the bandpasses with close mean wavelengths existing in different photometric systems. In Fig. 14 the positions and half-widths of bandpasses of the most popular medium-band photometric systems are 
shown. One can see that there is no problem to join some bandpasses of the uvby, the Geneva and the Vilnius photometric systems. Such an attempt to join the Vilnius and Geneva systems has been undertaken by Straižys, Jodinskienè and Hauck (1982) and by North, Hauck and Straižys (1982). The seven-colour system VilGen provides the same information as both original systems. At the same time it permits a shorter integration time or fainter limiting magnitude in comparison with the original Vilnius system. The joining of the VilGen system with the Strömgren system is also possible.

\section{References}

Boyle, R.P., Smriglio, F., Nandy, K. and Straižys, V. 1990a, A\&AS, 84, 1. Boyle, R.P., Smriglio, F., Nandy, K. and Straižys, V. 1990b, A\&AS, 86, 395.

Edvardsson, B. and Bell, R.A. 1989, MNRAS, 238, 1121.

Griffiths, R. 1985, Wide Field and Planetary Camera Instrument Handbook, Space Telescope Science Institute, Baltimore.

Griffiths, R. 1990, Wide Field and Planetary Camera Instrument Handbook, Version 2.1, Space Telescope Science Institute, Baltimore.

Harris, H.C., Baum, W.A., Hunter, D.A. and Kreidl, T.J. 1991, AJ, 101, 677.

Holtzman, J.A., Groth, E.J. et al. 1991, ApJ, 369, L35.

Hughes, S.M.G. 1992, in Stellar Photometry, ed. I. Eliott, IAU Colloq. No. 136, Dublin.

Koornneef, J., Bohlin, R., Buser, R., Horne, K. and Turnshek, D. 1986, in

Highlights of Astronomy, vol. 7, ed. J.-P. Swings, p. 833.

MacKenty, J.W., Griffiths, R.E., Sparks, W.B. et al. 1992, HST Wide

Field and Planetary Camera Instrument Handbook, Version 3.0, Space

Telescope Science Institute, Baltimore.

North, P., Hauck, B. and Straižys, V. 1982, A\&A, 108, 373.

Paltoglou, G. and Bell, R.A. 1991, MNRAS, 253, 449.

Smriglio, F., Nandy, K., Boyle, R.P., Dasgupta, A.K., Straižys, V. and Janulis, R. 1991, A\&AS, 88, 87.

Straižys, V. 1973, Bull. Vilnius Obs., No. 36, 3.

Straižys, V. 1977, Multicolor Stellar Photometry, Mokslas Publishers, Vilnius.

Straižys, V. 1992a, Multicolor Stellar Photometry, Pachart Publ. House, Tucson, Arizona.

Straižys, V. 1992b, Baltic Astron., 1, 107. 
Straižys, V., Jodinskienè, E. and Hauck, B. 1982, Bull. Vilnius Obs., No. 60,50 .

Straižys, V. and Sviderskienè, Z. 1972, A\&A, 17, 312.

Straižys, V. and Sviderskiené, Z. 1983, A\&SS, 94, 23.

Straižys, V. and Zdanavičius, K. 1970, Bull. Vilnius Obs., No. 29, 15.

Sviderskienè, Z. 1988, Bull. Vilnius Obs., No. 80, 3.

Trauger, J. 1989, in Highlights of Astronomy, vol. 8, ed. D. McNally, p. 443.

Trauger, J.T. and Brown, D.J. 1992, WFPC-2 Science Observation and Engineering Modes, Version 1.0, JPL.

Westphal, J.A. 1982, in The Space Telescope Observatory, Space Telescope

Science Institute, ed. D.N.B. Hall, p. 28. 